sous la lumière plus douce de l'automne vieillissant? N'y a-t-il pas aussi quelque chose d'émouvant dans l'effort vain de l'écorce pour protéger les moignons mis à nu par la taille?

C'est ce que pense un ami, hanté par les souvenirs, en méditant dans les vergers fleuris d'un vieux pays qui lui aurait plu par sa grâce et la violence même de ses contrastes.

Sierre, avril 1943.

G. Piotet.

\title{
Galilée et le problème du temps
}

Des voix plus autorisées que la mienne célébreront ici la gloire de Galilée. N'étant pas historien, je me garderai de décrire l'un ou l'autre des épisodes de cette vie admirable. Je voudrais plutôt, après tant d'autres, situer quelques-unes des découvertes de Galilée dans le cadre des progrès de la mécanique et caractériser de mon mieux l'étape qu'il a fait franchir à la science à l'aurore des temps modernes. Bien que l'étude sur la chute des corps ait précédé les découvertes astronomiques, c'est par ces dernières que nous commencerons.

Pour les disciples d'Aristote, si nombreux encore à l'époque de la Renaissance, l'univers se composait de deux parties nettement distinctes et qui étaient chacune régies par des principes propres. Le monde terrestre, dit aussi sublunaire, donc en deça de la lune, était celui des choses corruptibles; tandis qu'au delà se mouvaient les sphères parfaites le long des épicycles de Ptolémée. Les mouvements des astres ne pouvaient être que circulaires ou composés de mouvements circulaires uniformes en vertu même de la perfection de la circonférence, partout identique à elle-même. L'observation des mouvements des astres avait été poussée très loin durant l'antiquité, puis chez les Arabes, et enfin à l'époque qui précède celle de Galilée. Les périodes du mouvement des planètes, la période lunaire, le phénomène de la précession des équinoxes, l'angle variable que fait l'axe du monde avec celui de l'écliptique, avaient été mesurés avec une précision devant laquelle on reste confondu. Des tables astronomiques étaient établies, qui permettaient de prévoir les retours des astres et constituaient une excellente connaissance des temps, comme diraient les astronomes. Mais, de quoi s'agis- 
sait-il? essentiellement de la constatation des périodicités des phénomènes célestes, tels qu'ils apparaissent vus de la terre. On pouvait supposer la terre fixe et il fallait que le soleil, les planètes, toutes les étoiles, décrivissent en un jour leur mouvement autour de nous. Au XVI ${ }^{\mathrm{e}}$ siècle encore, le sens commun avait peine à imaginer une terre en mouvement. Les oiseaux, disait-on, ne retrouveraient plus leurs nids. Enfin, la destinée humaine exigeait que nous fussions placés au centre de tout.

Mais, bien entendu, d'autres opinions s'étaient déjà fait jour dès l'époque pythagoricienne. Philolaos, Aristarque de Samos, plus récemment Nicolas de Cusa soutenaient qu'il était plus simple de demander à notre habitat de tourner, plutôt qu'à l'ensemble de l'univers céleste et avaient admis déjà le mouvement annuel de la terre. En 1368, Albert de Saxe disait d'un de ses maîtres qu'il soutenait l'équivalence entre la rotation de la terre et celle de l'ensemble du ciel, et l'impossibilité de choisir entre les deux théories. Les deux conceptions, rotation de l'ensemble de la voûte céleste, ou rotation diurne de la terre, expliquaient toutes deux les mouvements apparents. Que ces deux théories aient pu subsister côte à côte dans les écoles scientifiques, est une manifestation éclatante d'une première forme très générale du principe de relativité des mouvements apparents. Il y avait mouvement relatif des astres et de la terre, sans que l'on puisse trancher et dire ce qui était immobile. Ici, ce principe était uniquement de l'ordre de la cinématique, c'està-dire relatif à la description des mouvements. Malheureusement, des raisons extra-scientifiques faisaient en général pencher la balance d'un côté ou de l'autre et ce n'est pas dans la sérénité d'une pensée scientifique objective que ce grand principe de la relativité du mouvement aurait pu être accepté.

Trois systèmes principaux avaient été construits. $1^{0}$ Le système de Ptolémée où la terre est fixe, le soleil et les planètes décrivant autour d'elle des trajectoires assez compliquées, mais où le soleil n'avait pas encore de place privilégiée. $2^{0}$ Le système de Copernic. Le centre est le soleil. La terre et toutes les planètes tournent autour de lui, et la terre en plus tourne sur elle-même. Le grand mérite de Copernic, qui reprenait certaines thèses de l'astronomie grecque, d'Aristarque en particulier, est d'avoir mis d'accord le système héliocentrique avec les observations plus précises qui avaient été faites entre-temps. Mais, pour Copernic, les trajectoires sont encore les cycles et épicycles de Ptolémée, circonférences centrées non forcément sur le soleil, mais sur des excentriques, 
qui coïncideraient à peu près avec les centres des ellipses keplériennes entrevues plus tard. $3^{0}$ Le système de Tycho-Brahe, système mixte et bizarre, mais légitime au point de vue de la seule description des mouvements apparents. La terre est fixe, comme pour Ptolémée. Le soleil tourne autour de la terre, et les planètes tournent autour du soleil, accompagnant ce dernier dans sa course.

Il ne s'agissait que d'obtenir des schémas simples, inspirés des mouvements circulaires et qui rendissent compte des mouvements apparents, tels qu'ils étaient connus à la Renaissance. Tant que l'on ne se faisait pas une juste idée des dimensions relatives de la terre et du soleil d'une part, du système solaire et du système des étoiles d'autre part, ces différentes conceptions pouvaient être maintenues.

Quant aux causes entrevues des mouvements célestes, elles étaient multiples, et si diverses suivant les écoles que je n'entreprendrai pas ici de les décrire. L'idée d'attraction se trouve incidemment chez Copernic, mais elle était loin d'être sérieusement prise en considération. Les astres, pour la plupart des savants et des philosophes, obéissaient davantage à des principes en quelque sorte esthétiques, réalisant l'harmonie des cercles et des sphères.

Le dispositif des lunettes d'approche paraît avoir été connu de Roger Bacon (1270), mais il fallait attendre le perfectionnement de la verrerie pour pouvoir en tirer un parti utile. En 1609, Galilée apprend qu'en Hollande on vient d'inventer un procédé pour grossir la vue au moyen de deux lentilles circulaires situées dans un même tube. Très rapidement il construit suivant ce principe un télescope d'un grossissement qui laisse bien en arrière les lunettes hollandaises. Il n'y eut pas dans l'histoire de l'astronomie de moment plus sensationnel que celui où Galilée dirigeait son télescope vers les différents corps célestes. L'homme, par sa technique, avait plus que triplé son sens le plus remarquable: la vue, qui l'avait déjà mis en rapport avec le firmament. L'observation simple allait-elle confirmer ou infirmer les idées communes? Allait-elle pouvoir trancher entre les grands systèmes philosophiques que l'on avait construits pour répondre à des exigences de toute sorte? La lune tout d'abord, bien loin d'être une sphère parfaite, est recouverte de montagnes plus hautes que celles de la terre; en plus, elle balance sur elle-même, mouvement dit de libration; elle nous présente tantôt un de ses bords tantôt un autre, bien qu'en gros ce soit toujours la même face que nous observions. Ce mouvement alternatif ou pendulaire dut paraître assez 
curieux aux disciples d'Aristote. L'astronome florentin aperçoit pour la première fois les taches solaires, puis la rotation du soleil sur lui-même, rotation qui se fait en une trentaine de jours. La matière des corps célestes n'était donc pas aussi incorruptible qu'on le pensait. Des accidents s'y manifestaient, premier rapprochement avec les contingences d'ici-bas. La distinction entre les deux mondes sublunaire et céleste allait s'affaiblissant. A l'époque de Tycho une comète était apparue, que dis-je une étoile nouvelle, le ciel était donc le théâtre d'apparitions nouvelles et de disparitions, d'engendrements et de décrépitudes. Et les planètes, qu'allaient-elles révêler? Vénus présentait des phases analogues à celles de la lune; elle peut être pleine, croissante ou décroissante, ou presque éteinte. Les positions relatives du soleil, de la terre et de Vénus pouvaient seules être la cause de ce phénomène, comme pour la lune. Les astronomes et Galilée lui-même virent dans les phases de Vénus la confirmation des idées de Copernic. Certes, cette constatation faisait pencher la balance en faveur du système de l'astronome polonais; mais cependant, l'argument n'était pas décisif et les phases de Vénus s'expliqueraient dans tout système où le mouvement relatif des trois corps soleil-Vénus-terre fût maintenu, dans celui de Tycho par exemple. L'argument tiré de Jupiter avait, semble-t-il, un tout autre poids. C'est une des premières observations de Galilée; elle date du 7 janvier 1610.

Galilée fut surpris d'apercevoir trois, puis quatre petits astres qui tournaient incontestablement autour de Jupiter avec une rapidité inouie; le plus rapproché, Io, fait le tour en un de nos jours, un autre en sept, la période allant s'allongeant avec la distance, comme le voulait Copernic en ce qui concerne le soleil et les planètes. Ces petits satellites de Jupiter, en tous cas, ne tournaient pas autour de la terre, ou s'ils le faisaient avec Jupiter, ils avaient pour cette planète une considération toute particulière. Le monde Jovien, tenant tout entier dans le champ de la lunette, avait en faveur de Copernic une très forte valeur analogique. Saturne présentait à l'époque, comme par pudeur, son anneau un peu trop de profil, si bien que l'astronome florentin ne put faire une description correcte de cette merveille de la nature.

Galilée observa, par la rotation du soleil, et la rotation des satellites de Jupiter, l'uniformité du sens de la rotation, dans le système solaire. On sait le parti qu'en tira Laplace pour son hypothèse cosmogonique; il est vrai que plus tard on s'aperçut que le monde uranien tourne en sens inverse sur lui-même. 
Et les étoiles? Le télescope ne les grossissait pas, ne les rapprochait pas! Résultat négatif, mais combien plus instructif encore que les autres. Leur grandeur apparente était nulle en face de leur éclat.

Quant à la voie lactée, elle se résorbait, même dans ses parties les plus denses, en une multitude de petites étoiles. Galilée a-t-il observé quelques-unes des grandes nébuleuses, celle d'Andromède en particulier, je ne sais; peut-être s'attendait-il là aussi à une résorption en un amas d'étoiles.

Galilée créateur de l'astrophysique ne croyait pas à la propagation instantanée de la lumière, Descartes plus tard l'affirmait encore. Il a rejeté les étoiles à des distances incommensurablement plus grandes que celle qui régnent à l'intérieur du système solaire. Il aurait déjà pu nous faire frissonner en employant le langage moderne des années lumière. Soleil, planètes et satellites formés d'une matière corruptible s'isolaient donc du reste de l'univers et paraissaient de plus en plus devoir évoluer suivant le schéma copernicien.

On ne saurait assez souligner la prudence de Galilée qui n'abandonnait les idées d'Aristote ou celles de Ptolémée que si cela devenait absolument nécessaire, qui ne fit pas de généralisation hâtive, d'extrapolation imprudente, tout au moins dans ses écrits, qui n'avoua se rallier au système de Copernic qu'à la fin de sa carrière. Sa condamnation fut d'autant plus navrante que son objectivité avait été plus manifeste.

Les travaux de Galilée sur la chute des graves sont tout aussi importants pour l'histoire de la pensée scientifique, quoique moins sensationnels que ses observations astronomiques.

C'est cette importance que je voudrais faire ressortir. Il faut de nouveau replacer l'astronome florentin dans le milieu créé par les travaux de ses devancier pour, ici encore, mieux dégager l'étape de Galilée dans l'histoire des sciences.

Pour les péripatéticiens, une vitesse dans le monde sublunaire ne pouvait subsister que si une force l'entretenait. La force en un mot créait la vitesse. Les graves tendaient à rejoindre leur lieu naturel qui est en bas. Un mouvement ascendant ne pouvait être entretenu que par une sorte de réaction environnante, par l'air ébranlé lors du lancement vers le haut. La physique était toute imprégnée de finalisme et de la doctrine des causes d'Aristote, qui étaient plus aptes à fournir les prémisses à des syllogismes purement grammaticaux qu'à suivre les phénomènes dans le détail de leur comportement. Mais, de nombreux savants à 
cette époque réclamaient une étude plus objective des phénomènes et, en ce qui concerne la chute des graves, Galilée a eu des précurseurs. Il ne fut pas le premier à mesurer les temps de chute et les espaces parcourus, ni en chute libre ni sur le plan incliné. Déjà Epicure avait affirmé que les corps tomberaient dans le vide avec la même vitesse quelle qu'en soit la nature, bille de bois ou bille de plomb. D'autres, parmi lesquels on peut citer Oresme et Léonard de Vinci, avaient réuni ce que l'on pourrait appeler les éléments statistiques de ce phénomène de la chute des corps.

Quel fut donc l'apport de Galilée qui entreprit dès l'âge de 25 ans des travaux sur ce sujet? Benedetti avait déjà introduit le concept d'accélération mais sous une forme vague. Galilée, dans ce domaine, apporte un esprit mathématique plus profond que ses précurseurs, et qui rappelle celui d'Archimède. Que de qualités devaient être réunies en un seul homme, pour dégager d'un phénomène si simple que la chute d'une pierre, un des éléments essentiels de ce que nous appelons aujourd'hui la physique mathématique, élément que j’essaierai de définir dans quelques minutes: conscience de l'expérimentateur scrupuleux, sagacité du logicien qui sait isoler une proposition et la rendre plausible, et surtout le génie mathématique, car le problème même relevait de l'analyse infinitésimale qui n'était pas encore inventée. Le mathématicien Lagrange dit dans son ouvrage de mécanique analytique: «Il fallait un génie extraordinaire pour démêler les lois de la nature dans des phénomènes que l'on avait toujours eu sous les yeux, mais dont l'explication avait néanmoins toujours échappé aux recherches des philosophes.»

En 1590, Galilée lâche deux billes de plomb de grosseur différente. Elles touchent le sol en même temps, tandis que les péripatéticiens soutenaient que la vitesse était proportionnelle au poids. Il était bien évident que les vitesses s'accroissent au cours de la chute, mais de ce renseignement qualitatif Galilée ne se contente pas, il veut savoir comment varie la vitesse au cours du temps. Il admet tout d'abord que la vitesse, nulle au départ, croît proportionnellement à l'espace parcouru. Cette hypothèse, il la rejette car elle serait contradictoire en elle-même; c'est une merveille qu'il l'ait aperçu avant que le calcul intégral fût créé; le point ne saurait se mettre en mouvement s'il devait en être ainsi. Puis il admet que la vitesse varie proportionnellement au temps de la chute. L'accroissement de la vitesse est alors proportionnel à celui du temps. Leur 
rapport est une constante : «Aucun accroissement, dit Galilée, ne nous paraît plus simple que celui qui se fait toujours de la même manière». C'est donc l'accélération qui est supposée constante et l'expérience vient confirmer cette hypothèse simple. Galilée montre par les ressources de l'analyse mathématique naissante, que si la vitesse croît comme le temps, les espaces parcourus croîtront comme les carrés des temps.

Galilée a nettement conçu le principe de réversibilité des phénomènes de la mécanique pure et vu que si le mobile était rejeté en sens inverse le long du plan incliné, avec la même vitesse qu'il a en un point, il remonterait jusqu'à la même hauteur comme une escarpolette en ayant en chaque point intermédiaire la même vitesse qu'il avait à la descente. Galilée en déduit cette autre proposition qui exprime, elle aussi, un théorème qui est un pressentiment du théorème de la conservation de l'énergie mécanique: "Les degrés de vitesse, dit-il, d'un mobile descendant, par un mouvement naturel, de la même hauteur, sur des plans inclinés d'une manière quelconque, sont toujours égaux à l'arrivée sur une même horizontale, quand tous les obstacles sont écartés.» Il a donc aperçu les différents théorèmes généraux qui interviennent dans le problème de la chute, les reliant entre eux et les confrontant avec l'expérience.

Il enchaîne par la force de la pensée mathématique infinitésimale tous les résultats de mesures faites par ses devanciers et par lui-même. Mais ces relations dépassaient le niveau mathématique de son temps et appartiennent aux champs du calcul différentiel et intégral. ${ }^{1}$

La partie mathématique de ces relations, suivant que l'on s'attache aux espaces, aux temps, aux vitesses, aux différentes pentes du plan incliné, ne fait aucune difficulté pour celui qui possède l'analyse infinitésimale, mais à l'époque, elle n'était ni inventée ni surtout poussée à ce point de perfection où elle est aujourd'hui, et cependant les écrits de Galilée sur la chute des graves ne paraissent pas vieilli, c'est au moins l'impression que j'en ai eu en lisant quelques pages de ses dialogues. Ils mettent aux prises trois personnages. Salviati - c'est Galilée lui-même —, Sagredo est un homme qui cherche à comprendre mais qui est réticent, presque pyrrhonien. C'est par sa bouche que des objections analogues à celles de Zénon d'Elée interviennent; vous ne sauriez diviser indéfiniment des

1 Au point de vue de l'astronomie mathématique, il ne serait pas exagéré de dire que l'arithmétique caractérise l'époque babylonienne, la géométrie l'époque grecque, la trigonométrie l'époque arabe, et enfin, l'analyse infinitésimale l'époque moderne. 
espaces parcourus ou des durées employées à les parcourir, vous n'arriverez jamais au bout et votre pierre ne démarrerait même pas si elle devait attendre que vous eussiez achevé vos opérations dichotomiques de l'espace et du temps. Sagredo introduit dans le débat la querelle des indivisibles qui devait tant troubler les écoles mathématiques de la Renaissance. Enfin, Simplicius feint de comprendre et finit par tout rejeter pour des raisons qui n'ont rien à voir avec les problèmes étudiés.

On entend dire souvent que les savants dégagent le «comment»des phénomènes et les philosophes le «pourquoi». Ce sont les philosophes qui pensent ainsi, bien entendu. Or Galilée dépasse en force et en prudence les disciples du Stagirite lorsqu'il dit ceci: «Il ne me paraît pas opportun de rechercher, pour le moment, quelle est la cause de l'accélération du mouvement naturel. Sur ce sujet, des opinions diverses ont été émises par divers philosophes; les uns l'ont attribuée au rapprochement du centre, les autres...». Descartes reprochait précisément à Galilée de n'avoir pas étudié la cause de la chute des corps. Les mésaventures de la science lorsqu'il s'agit de la cause de la gravitation nous font au contraire apprécier davantage la prudence de l'astronome de Florence qui, comme plus tard Newton, se refusait à dire le pourquoi de la gravitation.

Cependant il fondait solidement une des culées du pont qu'allait construire Newton entre les mécaniques terrestre et céleste. L'introduction des forces allait en même temps restreindre le principe de relativité et faire pencher la balance définitivement en faveur de Copernic. Bien souvent, dans la science, c'est, l'introduction d'un principe nouveau, ici la force, qui permet de trancher les alternatives.

Galilée est le premier physicien qui ait mesuré le coefficient de la pesanteur g; il ne doit pas être confondu avec le poids et est le même pour tous les corps. Ce coefficient caractérise donc la capacité qu'a la terre à faire tomber les corps; c'est ce que l'on appelle aujourd'hui une propriété du champ. Sans elle, le système de Newton et la mécanique céleste eussent été impossibles à édifier plus tard, car la trajectoire d'une planète pourrait alors dépendre de la nature de la matière de cette planète. On sait quel profit Einstein a tiré de cette remarque faite par Benedetti et Galilée, que les corps tombent avec la même vitesse dans le vide quel qu'en soit le poids. De propriété du champ physique, elle est devenue propriété de l'espace-temps. Passons au problème du temps, seconde partie de notre sujet. 
Les mouvements du soleil et des étoiles mesurent un temps astronomique et permettent de définir le jour et l'année. C'est aux périodicités des principaux phénomènes célestes qu'est dû le succès des prévisions astronomiques faites au cours de l'antiquité des chaldéens à la période alexandrine.

Ainsi le temps se mesurait à la grande horloge astronomique. Mais pour ce qui est des phénomènes terrestres et proches de nous, la science antique s'était restreinte à de la statique. Archimède, lui-même, s'était enfermé dans des études de mécanique où le temps n'intervient pas; comme le levier, ou les corps flottants. La science grècque n'a pas étudié les mouvements qui se déroulent sur la terre. Elle préférait l'immuable au changeant, l'immobile au mobile, linvariant à la transformation. ${ }^{1}$

La flèche de Zénon frémissait en volant au dessus du continu mathématique et bien des philosophes condamnaient le mouvement. L'immuable pour eux était seul un reflet de l'èternité.

La science grecque a négligé l'étude mathématique de l'évolution des systèmes matériels à la surface de la terre.

C'est à la renaissance, seulement, que l'on chercha à rapporter les phénomènes terrestres à l'écoulement du temps et à suivre leur évolution. L'astronomie pour employer une image, réglementait la vitesse d'écoulement d'un film cinématographique, mais aucun phénomène terrestre n’était projeté sur ce film.

Bergson a dit quelque part ceci qui me paraît particulièrement vrai: c'est la renaissance italienne qui a introduit le temps comme variable indépendante. Naturellement, cette entreprise exigeait la possibilité de mesurer des courtes périodes mieux que ne pouvaient le faire les sabliers ou les clepsydres. Galilée à 18 ans avait observé l'isochronisme des petites oscillations du pendule, sur un lampadaire du dôme de Pise, dit-on. Huygens allait en tirer parti pour construire de véritables horloges terrestres. La mesure précise du temps descendait du ciel sur la terre et l'expérimentateur eut, à partir de cette époque, un garde temps perfectionné.

C'est Léonard de Vinci, Galilée et quelques autres savants de la renaissance italienne qui les premiers ont étudié quantitativement l'évolution d'une expérience au cours du temps. Mais on peut reconnaître à Galilée un autre mérite encore. Il a introduit dans les équations de la mécanique

1 Sauf dans la physique toute qualitative et finaliste d'Aristote. 
l'accélération. En langage mathématique une accélération est une dérivée seconde. Or s'il est un fait avéré malgré toutes les révolutions de la physique, c'est que les équations qui expriment les grandes lois contiennent toujours au moins une dérivée seconde.

Galilée a le premier deviné cette forme des équations de la mécanique.

Il resterait beaucoup à dire et j'ai omis des choses essentielles que je ne ferai que de mentionner. Galilée a remarqué la propriété des mouvements de pouvoir s'ajouter. Ainsi, dans le problème du tir ou mouvement des projectiles, le mouvement réel est la résultante de la chute tel qu'elle serait suivant la verticale, et d'un mouvement uniforme horizontal. Galilée, Descartes et Huyggens sont les premiers à avoir formulé le principe d'inertie qui dit que la vitesse d'un corps restera toujours identique en direction et en grandeur, tant qu'aucune force ou aucun obstacle n'intervient.

La science relativiste contemporaine a reconnu le mérite de l'astronome florentin en donnant le nom de galiléen aux systèmes en translation rectiligne et uniforme, et le nom de transformation de Galilée, aux transformations des coordonnées d'espace et de temps lorsque l'on passe d'un tel système à un autre.

C'est pour les transformations de Galilée que les équations de la mécanique gardent leur forme, tandis que les équations de l'électromagnétisme gardent la leur pour la transformation de Lorentz-Einstein. Mais ce n'est pas une raison suffisante pour opposer Galilée aux modernes. Au contraire, nous voyons de la Renaissance à nos jours s'accomplir un progrès continu, quels que soient les énoncés particuliers de nos lois scientifiques, et c'est à Galilée que revient la gloire d'avoir créé quelques-unes des formes de pensée qui sont désormais inséparables de notre conception du monde.

Si les sciences politiques avaient fait autant de progrès que les sciences pures, nous n'aurions jamais calculé des paraboles de tir dans un but criminel. Mais les hommes sont multiples et ont des appétits matériels démesurés. Dans ces conditions, on peut se demander si l'idée de culture ou de civilisation n'est pas contradictoire, tant que subsistent chez l'homme de pareilles contradictions.

Admirons d'autant plus ceux qui ont su cultiver la science pour ellemême et la faire progresser, et Galilée est un des plus grands.

\section{Rolin Wavre.}

